\title{
Breakdown of Classical Nucleation Theory near Isostructural Phase Transitions
}

\author{
A. Cacciuto, S. Auer, ${ }^{*}$ and D. Frenkel \\ FOM Institute for Atomic and Molecular Physics, Kruislaan 407, 1098 SJ Amsterdam, The Netherlands
}

(Received 14 July 2004; published 15 October 2004)

\begin{abstract}
We report simulations of crystal nucleation in binary mixtures of hard spherical colloids with a size ratio of 1:10. The stable crystal phase of this system can be either dense or expanded. We find that, in the vicinity of the solid-solid critical point where the crystallites are highly compressible, small crystal nuclei are less dense than large nuclei. This phenomenon cannot be accounted for by either classical nucleation theory or by the Gibbsian droplet model. We argue that the observed behavior is due to the surface stress of the crystal nuclei. The observed effect highlights a general deficiency of the most frequently used thermodynamic theories for crystal nucleation. Surface stress should lead to an experimentally observable expansion of crystal nuclei of colloids with short-ranged attraction and of globular proteins.
\end{abstract}

DOI: 10.1103/PhysRevLett.93.166105

PACS numbers: 68.35.Md, 05.10.-a, 05.70.Np

The pathway for crystal nucleation can be strongly influenced by the presence of metastable phases. This observation dates back to Ostwald, who formulated his famous "step" rule stating that the crystal phase that nucleates from the melt need not be the one that is thermodynamically most stable, but the one that is closest in free energy to the parent phase [1]. Recent simulations $[2,3]$ and density-functional theory [4,5] provide an illustration on a microscopic scale that the vicinity of metastable phases may determine the properties of microscopic crystal nuclei. The relevant metastable phases need not be crystalline. For example, the presence of a critical demixing transition in the metastable liquid parent phase may have a dramatic effect on the nucleation process [3]. This scenario may be relevant for crystal nucleation in solutions of proteins or colloids, or in liquid metal alloys.

Virtually nothing is known about the nucleation pathway for cases where the crystal phase that nucleates is close to a solid-solid critical point. Such situations can arise in the nucleation close to the critical point of an isostructural solid-solid transition. Isostructural solidsolid transitions are expected to occur in crystalline alloys near a substitutional order-disorder transition or in systems of hard colloidal particles with a short-ranged attraction [6-9]. Here we consider the latter case. Depending on the range of attraction, the solid-solid critical point may either be located in a stable or in a metastable part of the phase diagram. Simulations by Dijkstra indicate that this is the case for mixtures of large and small hard colloids [9]. The small colloids (diameter $\sigma_{s}$ ) induce an effective attraction between the large colloids (diameter $\sigma_{l}$ ). The range of the attraction is determined by the size of the small colloids. For a size ratio $q \equiv\left(\sigma_{s} / \sigma_{l}\right)=0.05$, the phase diagram exhibits a stable isostructural critical point. For $q=0.1$ the range of attraction is longer and the isostructural critical point moves to the metastable region beyond the melting curve (see Fig. 1).

We performed Monte Carlo simulations to investigate how the presence of a metastable critical point in the crystal phase affects the early stages of the nucleation process.

The direct numerical simulation of a highly asymmetric hard-sphere mixture is computationally demanding [10]. However, for $q$ sufficiently small, we can account for the effect of the small spheres by using an effective potential $[9,11]$. The range and strength of this interaction depend on the size and concentration of the small spheres. The effective pair interaction between a pair of large colloids in contact with a reservoir of small spheres at volume fraction $\eta_{s}^{r}$ is approximately given by

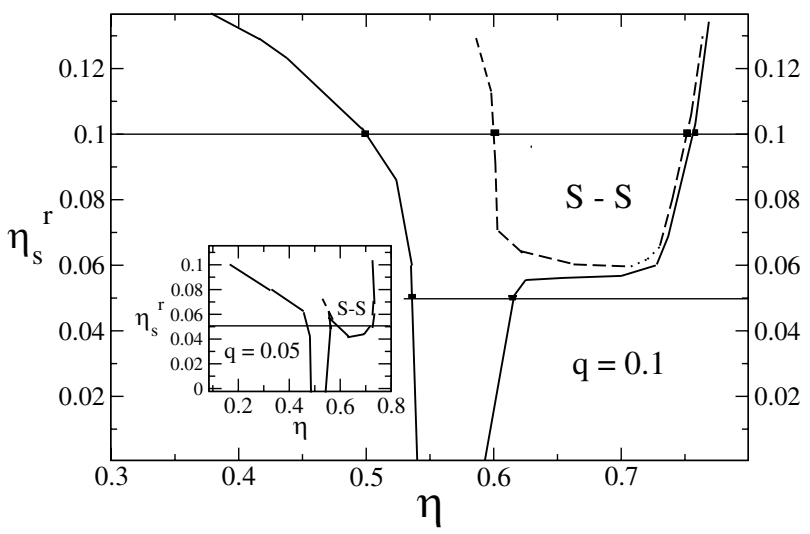

FIG. 1. Sketch of the phase diagram of a binary hard-sphere mixture of volume fraction $\eta$ for $q=0.1$ and $q=0.05$ (inset) obtained from data reported in Ref. [9]. $\eta_{s}^{r}$ is the volume fraction of a reservoir of small spheres in contact with the system. The dashed curves indicate the metastable solid-solid phase. Horizontal lines mark the values of $\eta_{s}^{r}$ where we performed simulations. 


$$
\beta \phi_{\mathrm{eff}}(R)= \begin{cases}-\frac{1+q}{2 q}\left[3 \lambda^{2} \eta_{s}^{r}+\right. & \left(R<\sigma_{l}+\sigma_{s}\right), \\ \left(9 \lambda+12 \lambda^{2}\right)\left(\eta_{s}^{r}\right)^{2}+ & \\ \left.\left(36 \lambda+30 \lambda^{2}\right)\left(\eta_{s}^{r}\right)^{3}\right], & \\ 0, & \left(R>\sigma_{l}+\sigma_{s}\right),\end{cases}
$$

where $R$ is the distance between the large colloids, $\lambda \equiv$ $R / \sigma_{s}-1 / q-1$ and $\beta \equiv 1 / k_{B} T$. We focus on small values of $\eta_{s}^{r}$ where it is permissible to ignore higher order corrections to this expansion [11].

We performed constant-pressure Monte Carlo simulations of binary hard-sphere mixture with a size ratio $q=$ 0.1 at two different values of $\eta_{s}^{r}$. For $\eta_{s}^{r}=0.05$, there is only one crystal phase. For $\eta_{s}^{r}=0.1$, a metastable crystal phase exists between the fluid phase and the equilibrium crystal phase (see Fig. 1).

We started from a stable liquid suspension of $N=3375$ large colloids and compressed it beyond the freezing density. We then computed $\Delta G$, the free-energy barrier for crystal nucleation, as a function of the size $n$ of the crystal nucleus (see [12] for technical details). This calculation was repeated at different supersaturations $\Delta \mu=$ $\left|\mu_{\text {sol }}-\mu_{\text {liq }}\right|$ (where $\mu_{\text {sol }}\left(\mu_{\text {liq }}\right)$ are the chemical potentials of the solid and liquid phases, respectively).

Figure 2 shows the computed crystal-nucleation barriers as a function of nucleus size $n$ for different values of $\Delta \mu$. Figure 3 shows how the height of the nucleation barrier $\Delta G^{*}$ depends on supersaturation. classical nucleation theory (CNT) [13] predicts that, at constant supersaturation, $\Delta G^{*}$ depends strongly on the solid-liquid interfacial free-energy density $\gamma_{l s}$ :

$$
\Delta G^{*} \sim\left(\gamma_{l s}^{3} / \Delta \mu^{2}\right)
$$

One should expect that $\gamma_{l s}$ increases with the strength of the effective attraction between the large colloids [14], and hence with the concentration of the small spheres. Hence $\Delta G^{*}$ should increase with $\eta_{s}^{r}$. This is precisely what is observed in Fig. 3.

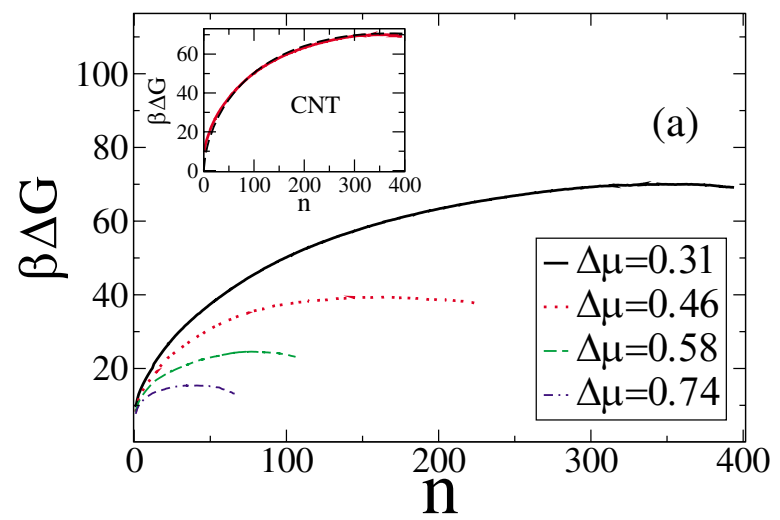

It is instructive to compare the shape of the computed nucleation barriers with the functional form predicted by classical nucleation theory (CNT) [13]):

$$
\Delta G=\gamma_{l s} S-|\Delta \mu| \rho_{s} V,
$$

where $S$ is the surface area of the crystal nucleus, $V$ is its volume, and $\rho_{s}$ is the density of the crystal at coexistence. Figure 2 shows that there is a striking difference in behavior between the systems with and without a metastable solid phase: in the absence of a metastable solid phase [Fig. 2(a)], classical nucleation theory accounts well for the shape of the nucleation barrier. But in the presence of a metastable solid phase [Fig. 2(b)], CNT cannot reproduce the shape of the nucleation barrier. One of the central assumptions of CNT is that the structure of the crystal nucleus is that of the stable bulk phase at coexistence. In the presence of a metastable solid phase, this assumption is violated. This is most easily seen by considering the density of crystal nuclei as a function of their size.

As there is some ambiguity in the definition of $\rho_{c}$, the number density of a small crystallite, we computed this quantity in three different ways. A rough estimate can be obtained by locating the center of mass of the cluster and then counting the number of particles inside spheres of increasing radius. A second estimate is obtained by matching the distribution of the nearest-neighbors distances inside a nucleus to that of a bulk crystal. The density of the bulk crystal that gives the best match defines $\rho_{c}$. A third approach was to perform a Voronoi construction around each particle inside the cluster. The density was then defined as the number of particles inside the nucleus divided by the sum of the volumes of the Voronoi polyhedra. Figure 4 shows the density of crystal nuclei of increasing size at $\eta_{s}^{r}=0.1$ and $\Delta \mu=0.65$. The densities were computed using the three methods described above and the difference between the results for different methods falls within the error bars. For each size, we considered a sample of 100 different clusters.

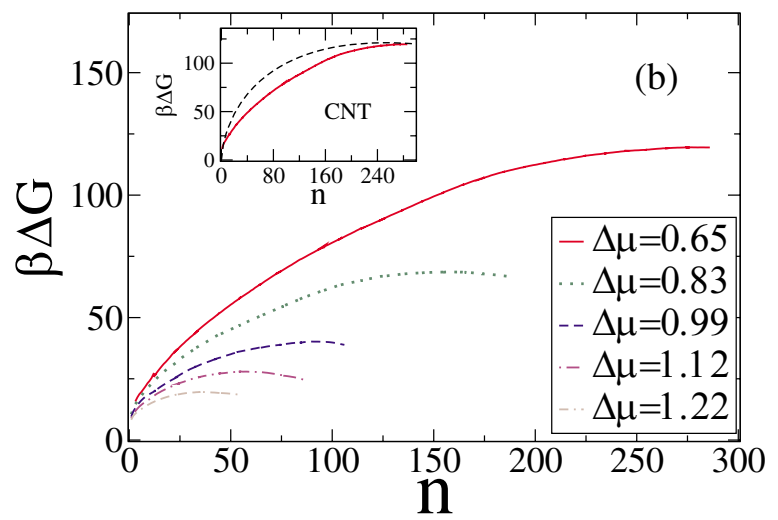

FIG. 2 (color online). Free-energy crystal-nucleation barriers $\Delta G(n)$ as a function of the nucleus size for $q=0.1$ at (a) $\eta_{s}^{r}=0.05$ and (b) $\eta_{s}^{r}=0.1$ for different values of the chemical potential. Error bars on $\Delta G$ are of the order of $1 k_{B} T$. The inset shows a comparison between the highest computed barrier and form predicted by CNT. 


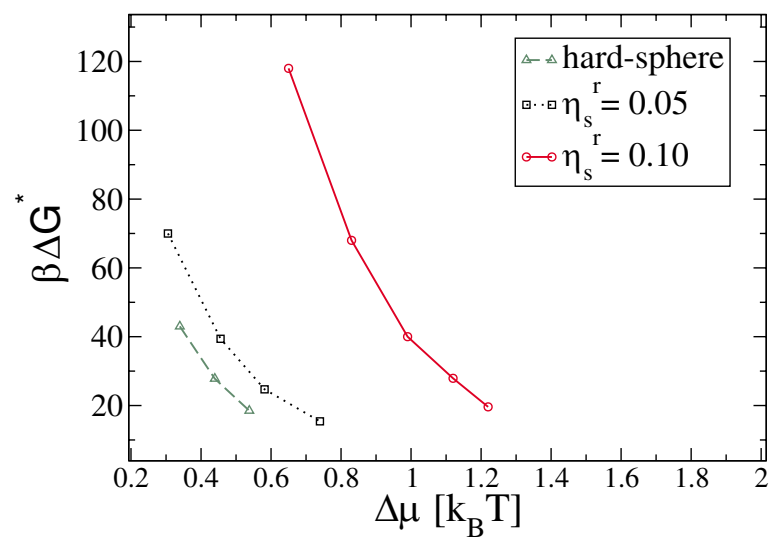

FIG. 3 (color online). Nucleation barrier height $\Delta G^{*}$ as a function of supersaturation $\Delta \mu$ for a system of hard spheres [20] and a binary mixtures with $q=0.1$ at $\eta_{s}^{r}=0.05$ and $\eta_{s}^{r}=$ 0.1 . Error bars on $\Delta G^{*}$ are of the order of $1 k_{B} T$.

The figure shows that the density of clusters increases with size. To rule out that the observed density change is a trivial finite-size effect, we repeated the same calculation for crystal nuclei at $\eta_{s}^{r}=0.05$ where no metastable solid phase is present. The inset of Fig. 4 shows that, in that case, the density of the crystal nuclei is effectively independent of size. Moreover, for mixtures with a size ratio $q=0.05$ (short-ranged effective interaction) the lowdensity solid phase becomes stable [9] near $\eta_{s}^{r}=0.05$, while the high-density solid phase is metastable (see Fig. 1). In that case, we always found that the crystal nuclei had densities corresponding to the low-density phase.

At the pressures for which we performed the simulations, the dense solid is, in the bulk, always more stable than the metastable expanded solid. It may therefore seem

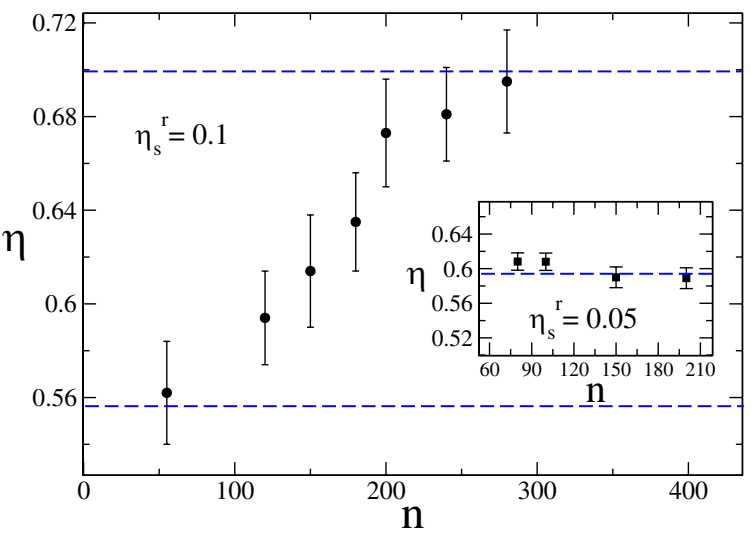

FIG. 4 (color online). Crystal clusters volume fraction as a function of their size for a binary mixture with $q=0.1$ and $\eta_{s}^{r}=0.1$ at $\Delta \mu=0.65$. The dashed lines indicate the location of the metastable solid-solid phase. The critical nucleus contains approximately $n \sim 290$ particles. The inset graphs the quantities for $\eta_{s}^{r}=0.05$ at $\Delta \mu=0.31$. Error bars are large enough to include the estimate obtained with the three methods described in the text. surprising to find that some crystal nuclei have a density close to that of the expanded solid phase. It is doubly surprising that it is the small crystal nuclei that have the lower density. In the Gibbsian droplet model of (liquidvapor) nucleation, the free-energy barrier for nucleation is computed by considering the effect of the Laplace pressure on the chemical potential of the critical nucleus. As the critical nucleus is in (unstable) equilibrium with the parent phase, it must have the same chemical potential. This is possible if the pressure inside the nucleus $\left(P_{n}\right)$ is higher than the pressure $P_{p}$ of the parent phase, such that $\mu_{n}\left(P_{n}\right)=\mu_{p}\left(P_{p}\right)$. In the droplet model, the pressures difference $\Delta P=P_{n}-P_{p}$ is assumed to be equal to the Laplace pressure $2 \gamma / r_{s}$, where $r_{s}$ is radius of the surface of tension. Assuming that we know $\gamma$ and the pressure dependence of the chemical potential of both phases, one can then compute $r$, the radius of the surface of tension of the critical nucleus, and the nucleation barrier itself $\left(4 \pi \gamma r^{2} / 3\right)$. In contrast to CNT, the droplet model allows for the compressibility of the phase that nucleates. As the Laplace pressure is always positive, the droplet model predicts that small nuclei are denser than the corresponding bulk phase. But as Gibbs already realized [15], the droplet model cannot be applied to crystallites because, the excess pressure of crystals is not equal to $2 \gamma / r_{s}$. For solid interfaces, one should distinguish between the surface tension and the surface stress. The correct expression for the excess pressure inside a critical crystal nucleus of density $\rho$ and radius $r$ is (see, e.g., Ref. [16]):

$$
\Delta P=\frac{2 \gamma-3 \rho\left(\frac{\partial \gamma}{\partial \rho}\right)}{r} .
$$

In the present case (hard spheres with short-ranged attraction), the derivative $\partial \gamma / \partial \rho$ is large and positive. So much so that $\Delta P$ can become negative. The theoretical analysis of Mullins [16] shows that, to lowest order in the crystal strain, the density change of a crystalline nucleus is given by

$$
\frac{\rho-\rho_{0}}{\rho_{0}}=-\left(\frac{\partial \gamma}{\partial \rho}\right)_{0} \frac{3 \rho_{0}}{r B},
$$

where $B$ is the bulk modulus of the solid. To linear order in the strain, the radius of the critical nucleus is related to the supersaturation through

$$
r=\frac{2 \gamma}{P_{0}^{S}(\mu)-P^{L}(\mu)},
$$

where $P_{0}^{S}(\mu)$ and $P^{L}(\mu)$ are the pressures of the bulk solid and of the metastable liquid at chemical potential $\mu . \gamma$ is the surface free-energy density of the strained nucleus. Note that $\Delta \rho$ is negative: hence the solid indeed expands. This expansion is inversely proportional both to the radius of the nucleus and to the bulk modulus of the crystal. As $B$ vanishes at the solid-solid critical point, the value of $B$ in Eq. (3) can be small for nuclei that form close to this 


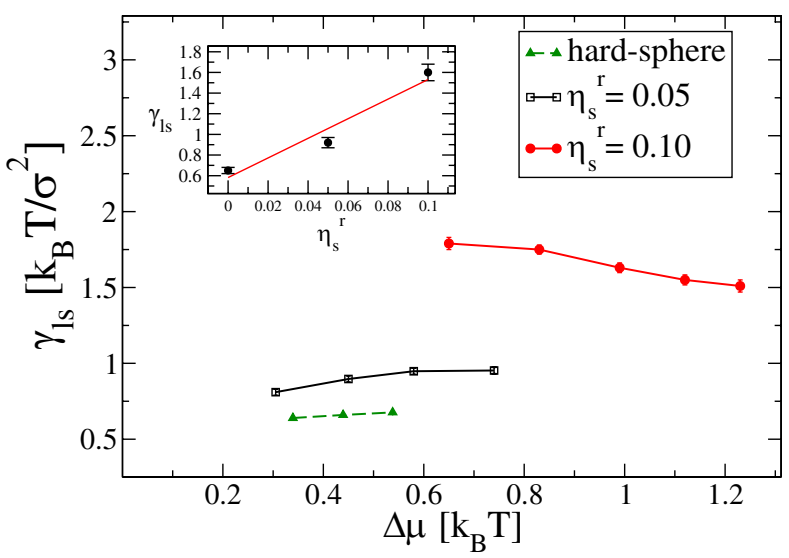

FIG. 5 (color online). Interfacial free-energy density as a function of supersaturation $\Delta \mu$ for a system of hard spheres [20,21] and a binary mixture with $q=0.1$ at $\eta_{s}^{r}=0.05$ and $\eta_{s}^{r}=0.1$. The inset shows the surface tension as a function of $\eta_{s}^{r}=0.1$. In the latter case, $\gamma$ decreases with increasing supersaturation. This is compatible with the thermodynamic analysis of Ref. [16]. The inset shows the surface tension as a function of $\eta_{s}^{r}=0.1$.

critical point. The expansion of the nucleus can therefore be large, in particular, for small $r$. This is precisely what we observe in the simulations. The observed expansion of small nuclei is in stark contrast with the predictions of both CNT and the droplet model.

Interestingly, the Gibbs expression for the height of the nucleation barrier is still valid, and this allows us to compute the surface free-energy density $\gamma$ of the strained nucleus from

$$
\gamma^{3}=\frac{3 \Delta G^{*}}{16 \pi}\left[P_{0}^{S}(\mu)-P_{L}(\mu)\right]^{2} .
$$

The resulting values of $\gamma$ are shown in Fig. 5. Finally, we can estimate the effect of surface stress on the excess pressure inside the nucleus. To lowest order in the strain, the strain-induced pressure change $\Delta P$ is given by

$$
\Delta P=\frac{B\left(\rho-\rho_{0}\right)}{\rho_{0}} .
$$

This is a large correction. For the small, expanded nuclei that we observe, $\Delta P / P_{L} \sim-20$. In the vicinity of a solidsolid transition, higher powers of the strain should be included to obtain a more accurate estimate of the straininduced pressure change. We have not attempted this here: our objective was simply to show that surface stress has a large effect on the internal pressure of crystal nuclei. The effect of surface stress on the density of crystal nuclei should be experimentally observable in the nucleation of compressible crystals, such as colloids with short-ranged attraction and globular proteins. Such an expansion can be determined by application of the nucleation theorem $[17,18]$ to the temperature or (osmotic) pressure dependence of the nucleation rate.
In summary, our simulations illustrate both quantitative and qualitative failures of CNT and the droplet model in the case of crystal nucleation. For crystals that are less compressible, the effect becomes small-but it is always there. Even for vapor-liquid nucleation there may be residual surface-stress effects in small droplets. This may explain the puzzling inconsistencies between the thermodynamical surface of tension and the mechanical surface of tension, as observed in simulations [19].

A. C. thanks I. Coluzza for helpful suggestions. The work of the FOM Institute is part of the research program of FOM and is made possible by financial support from the Netherlands organization for Scientific Research (NWO) and by Contracts No. NWO-04-016-022 and No. EU-MRTN-CT-2003-504712.

*Present Address: Department of Chemistry, Cambridge University, Lensfield Road, Cambridge, CB2 1EW, UK.

[1] W. Ostwald, Z. Phys. Chem. 22, 289 (1897).

[2] PR. ten Wolde, M. J. Ruiz Montero, and D. Frenkel, Phys. Rev. Lett. 75, 2714 (1995).

[3] P. R. ten Wolde and D. Frenkel, Science 277, 1975 (1997).

[4] V. Talanquer and D.W. Oxtoby, J. Chem. Phys. 109, 223 (1998).

[5] Y.C. Shen and D.W. Oxtoby, Phys. Rev. Lett. 77, 3585 (1996).

[6] P. Bolhuis and D. Frenkel, Phys. Rev. Lett. 72, 2211 (1994).

[7] A. Daanoun, C. F. Tejero, and M. Baus, Phys. Rev. E 50, 2913 (1994).

[8] C. N. Likos, Z.T. Nemeth, and H. Lowen, J. Phys. Condens. Matter 6, 10965 (1994).

[9] M. Dijkstra, R. van Roij, and R. Evans, Phys. Rev. E 59, 5744 (1999); Phys. Rev. Lett. 81, 2268 (1998).

[10] T. Biben, P. Bladon, and D. Frenkel, J. Phys. Condens. Matter 8, 10799 (1996).

[11] B. Götzelmann, R. Evans, and S. Dietrich, Phys. Rev. E 57, 6785 (1998).

[12] S. Auer and D. Frenkel, J. Chem. Phys. 120, 3015 (2004).

[13] K. F. Kelton, Crystal Nucleation in Liquids and Glasses, Solid State Physics Vol. 45 (Academic Press, Inc., New York, 1991), p. 75.

[14] R. P. Sear and D. Frenkel, Phys. Rev. Lett. 90, 195701 (2003).

[15] The Scientific Papers of J. Willard Gibbs (Ox Bow Press, Woodbridge, CT, 1993), Vol. 1.

[16] W.W. Mullins, J. Chem. Phys. 81, 1436 (1984).

[17] Y. Viisanen, R. Strey, and H. Reiss, J. Chem. Phys. 99, 4680 (1993).

[18] D.W. Oxtoby and D. Kashchiev, J. Chem. Phys. 100, 7665 (1994).

[19] P. R. ten Wolde and D. Frenkel, J. Chem. Phys. 109, 9901 (1998).

[20] S. Auer and D. Frenkel, Nature (London) 409, 1020 (2001).

[21] A. Cacciuto, S. Auer, and D. Frenkel, J. Chem. Phys. 119, 7467 (2003). 